\title{
ITERATIVE METHOD FOR SOLVING THE SECOND BOUNDARY VALUE PROBLEM (BVP) FOR BIHARMONIC TYPE EQUATION*
}

\author{
DANG QUANG A
}

\begin{abstract}
Solving BVPs for the fourth order differential equations by the reduction of them to BVPs for the second order equations with the aim to use the achievements for the latter ones attracts attention from many researchers.

In this paper, using the technique developed by ourselves in recent works, we construct iterative method for the second BVP for biharmonic type equation. The convergence rate of the method is established.
\end{abstract}

\section{INTRODUCTION}

Solving BVPs for the fourth order differential equations by the reduction of them to BVPs for the second order equations with the aim to use a lot of efficient algorithms for the latter ones attracts attention from many researchers. Namely, for the biharmonic equation with the Dirichlet boundary condition, there is intensively developed the iterative method, which leads the problem to two problems for the Poisson equation at each iteration (see e.g. $[3,8,9,11]$ ).

Recently, Abramov and Ulijanova [1] proposed an iterative method for the Dirichlet problem for the biharmonic type equation, but the convergence of the method is not proved. In our previous works $[5,6]$ with the help of boundary or mixed boundary-domain operators appropriately introduced, we constructed iterative methods for biharmonic and biharmonic type equations associated with the Dirichlet boundary condition. It is proved that the methods are convergent with the rate of geometric progression.

In this paper we develop our technique in [4-6] for the second BVP for the biharmonic type equation. Namely, we consider the following problem.

$$
\begin{aligned}
\Delta^{2} u-a \Delta u+b u & =f(x), x \in \Omega, \\
\left.\Delta u\right|_{\Gamma} & =g, \\
\left.u\right|_{\Gamma} & =h,
\end{aligned}
$$

where $\Delta$ is the Laplace operator, $\Omega$ is a bounded domain in $R^{n}(n \geq 2), \Gamma$ is the sufficiently smooth boundary of $\Omega, a$ and $b$ are constants. This problem has not yet considered in [1].

It should be noted that when $a=0, b=0$ Problem (1.1) $-(1.3)$ is decomposed into two consecutive problems for the Poisson equation. Otherwise, in the case, if one of the two coefficients $a$ and $b$ is negative, as mentioned in [2], Problem (1.1)-(1.3) is approximated by a 13-point difference scheme and a module for the latter problem is constructed. In [7] the problem in a rectangle, where $a=0, b>0$, is reduced to a system of two boundary integral equations and then is solved by the use of the Galerkin and collocation methods.

The present work is devoted to the solution of Problem (1.1)-(1.3), where $a \geq 0, b \geq 0$ and $a+b>0$. Two different cases will be treated in dependence on the sign of $a^{2}-4 b$.

\footnotetext{
* This work is supported in part by the National Basic Research Program in Natural Sciences, Vietnam.
} 


\section{CASE $a^{2}-4 b \geq 0$}

In this case we always can lead the original problem (1.1)-(1.3) to two problems for Helhomn equation. To do this, as in [5], we put

$$
\mu=\frac{1}{2}\left(a+\sqrt{a^{2}-4 b}\right) .
$$

Then Problem (1.1)-(1.3) is reduced to the following problems

$$
\begin{aligned}
\mu \Delta v-b v & =f, \quad x \in \Omega, \\
\left.v\right|_{\Gamma} & =\frac{1}{\mu} g-h, \\
\frac{1}{\mu} \Delta u-u & =v, \quad x \in \Omega, \\
\left.u\right|_{\Gamma} & =h .
\end{aligned}
$$

\section{CASE $a^{2}-4 b<0$}

This case is very important in mechanics, especially when $a=0, b>0$, because then we have the governing equation of a plate on elastic foundation (see [14]).

\subsection{Construction of iterative method}

As in [6], we set

$$
\begin{aligned}
& \Delta u=v, \\
& D=-b u .
\end{aligned}
$$

Then Problem (1.1)-(1.3) is led to the following two second order problems

$$
\begin{aligned}
\Delta v-a v & =f+D, \quad x \in \Omega, \\
\left.v\right|_{\Gamma} & =g, \\
\Delta u & =v, \quad x \in \Omega, \\
\left.u\right|_{\Gamma} & =h,
\end{aligned}
$$

where all the functions $u, v$ and $D$ are unknown but they are related with each other by (3.2).

To solve the above problems we use the following iterative process:

Step 1. Given a starting approximation $D^{(0)}$.

Step 2. Knowing $D^{(k)}(k=0,1, \ldots)$ solve consecutively two problems

$$
\begin{aligned}
\Delta v^{(k)}-a v^{(k)} & =f+D^{(k)}, x \in \Omega, \\
\left.v^{(k)}\right|_{\Gamma} & =g, \\
\Delta u^{(k)} & =v^{(k)}, \quad x \in \Omega, \\
\left.u^{(k)}\right|_{\Gamma} & =h .
\end{aligned}
$$

Step 3. Compute the new approximation

$$
D^{(k+1)}=D^{(k)}-\tau_{k+1}\left(D^{(k)}+b u^{(k)}\right), x \in \Omega,
$$

where $\tau_{k+1}$ are iterative parameters to be determined afterwards. 


\subsection{Investigation of convergence}

In order to investigate the convergence of the iterative process (3.5)-(3.7), firstly we rewrite (3.7) in the canonical form of two-layer iterative scheme $[12,13]$

$$
\frac{D^{(k+1)}-D^{(k)}}{\tau_{k+1}}+\left(D^{(k)}+b u^{(k)}\right)=0 .
$$

Now, we introduce the operator $B$ defined by the formula

$$
B D=D+b u
$$

where $u$ is the function determined from the problems

$$
\begin{aligned}
\Delta v-a v & =D, \quad x \in \Omega, \\
\left.v\right|_{\Gamma} & =0, \\
\Delta u & =v ; \quad x \in \Omega \\
\left.u\right|_{\Gamma} & =0,
\end{aligned}
$$

The properties of the operator $B$ will be investigated in the sequel.

Next, in Problems (3.3) and (3.4) we put

$$
u=u_{1}+u_{2}, \quad v=v_{1}+v_{2},
$$

where $u_{1}, u_{2}, v_{1}, v_{2}$ are the solutions of the problems

$$
\begin{gathered}
\Delta v_{1}-a v_{1}=D, \quad x \in \Omega,\left.\quad v_{1}\right|_{\Gamma}=0, \\
\Delta u_{1}=v_{1}, \quad x \in \Omega,\left.\quad u_{1}\right|_{\Gamma}=0, \\
\Delta v_{2}-a v_{2}=f, \quad x \in \Omega,\left.\quad v_{2}\right|_{\Gamma}=g, \\
\Delta u_{2}=v_{2}, \quad x \in \Omega,\left.\quad u_{2}\right|_{\Gamma}=h .
\end{gathered}
$$

From (3.12) and (3.13) by the definition of $B$ we have

$$
B D=D+b u_{1}
$$

We also see that (3.14) and (3.15) define an unique function $u_{2}$.

Since the solution $u$ found (3.3) and (3.4) should satisfy the relation (3.2), we have

$$
D+b\left(u_{1}+u_{2}\right)=0 \text {. }
$$

Hence, from (3.16) it follows

$$
B D=F,
$$

where

$$
F=-b u_{2} \text {. }
$$

We see that (3.18) is an operator equation for $D$, where the right-hand side $F$ is completely defined by the given functions $f, g, h$ of Problem (1.1)-(1.3). 
Proposition 1. The iterative process (3.5)-(3.7) is a realization of the two-layer iterative scheme

$$
\frac{D^{(k+1)}-D^{(k)}}{\tau_{k+1}}+B D^{(k)}=F, k=0,1,2, \ldots
$$

for the operator equation ( 3.18$)$.

Proof. Indeed, if in (3.5)-(3.7) we put

$$
u^{(k)}=u_{1}^{(k)}+u_{2}, v^{(k)}=v_{1}^{(k)}+v_{2}
$$

where $u_{2}, v_{2}$ are solutions of Problems (3.14), (3.15), then we get

$$
\begin{gathered}
\Delta v_{1}^{(k)}-a v_{1}^{(k)}=D^{(k)}, \quad x \in \Omega, \\
\left.v_{1}^{(k)}\right|_{\Gamma}=0, \\
\Delta u_{1}^{(k)}=v_{1}^{(k)}, \quad x \in \Omega, \\
\left.u_{1}^{(k)}\right|_{\Gamma}=0 .
\end{gathered}
$$

From here it is easy to see that

$$
B D^{(k)}=D^{(k)}+b u_{1}^{(k)} .
$$

Taking into account (3.19) we obtain

$$
D^{(k)}+b u^{(k)}=D^{(k)}+b u_{1}^{(k)}+b u_{2}^{(k)}=B D^{(k)}-F .
$$

Consequently, the scheme (3.8) may be written in the form of (3.20).

Thus, the proposition is proved.

Proposition 1 enables us to lead the investigation of convergence of Process (3.5)-(3.7) to the study of the scheme (3.20).

For this reason we need some properties of the operator $B$.

Proposition 2. The operator $B$ defined by (3.9)-(3.11) is linear, symmetric positive definite and bounded in the space $L_{12}(\Omega)$ of quadratic integralable functions in $\Omega$.

Proof. The linearity of $B$ is obvious. To establish the other properties of $B$ let us consider the inner product $(B D, \bar{D})$ of two arbitrary functions $D$ and $\bar{D}$ in $L_{2}(\Omega)$. We have

$$
(B D, \bar{D})=(D+b u, \bar{D})=(D, \bar{D})+b \int_{\Omega} u \bar{D} d x
$$

Here $u$ is found from (3.10)-(3.11). We also denoted by $\bar{u}$ and $\bar{v}$ the solution of (3.10)-(3.11), where $D$ is replaced by $\bar{D}$.

Now, we compute the integral in right-hand side of (3.25), which is denoted by $J$. We have

$$
\begin{aligned}
J=\int_{\Omega} u \bar{D} d x & =\int_{\Omega} u(\Delta \bar{v}-a \bar{v}) d x=\int_{\Omega} u \Delta \bar{v} d x-\int_{\Omega} a u \bar{v} d x \\
& =\int_{\Omega} u \Delta \bar{v} d x-a \int_{\Omega} u \Delta \bar{u} d x
\end{aligned}
$$


Applying the Green formula for functions vanishing on boundary, we obtain

$$
J=\int_{\Omega}(v \bar{v}+a \operatorname{grad} u \cdot \operatorname{grad} \bar{u}) d x .
$$

Hence,

$$
(B D, \bar{D})=\int_{\Omega}(D \bar{D}+v \bar{v}+a \operatorname{grad} u \cdot \operatorname{grad} \bar{u}) d x .
$$

Clearly,

$$
(B D, \bar{D})=(B, \bar{D}, D)
$$

and

$$
\left(B D, D=\int_{\Omega}\left(D^{2}+v^{2}+a|\operatorname{grad} u|^{2}\right) d x \geq(D, D) .\right.
$$

Thus, $B$ is symmetric and positive definite in $L_{2}(\Omega)$.

Further, from the theory of BVPs in [10], for the solutions $u$ and $v$ of Problems (3.10), (3.11) we have the estimates

$$
\begin{aligned}
\|v\|_{H^{2}(\Omega)} & \leq \sqrt{C_{1}}\|D\|_{L_{\gamma^{2}}(\Omega)} \\
\|u\|_{H^{2}(\Omega)} & \leq \sqrt{C_{2}}\|v\|_{L_{2}(\Omega)}^{v}
\end{aligned}
$$

where $C_{1}$ and $C_{2}$ are positive constants, $H^{s}(\Omega)$ is the Sobolev space.

Using these estimates it is easy to get the following estimate

$$
(B D, D) \leq\left(1+C_{2}+a C_{1} C_{2}\right)(D, D),
$$

which implies the boundness of $B$ in $L_{2}(\Omega)$.

Thus, the proof of Proposition 2 in complete.

In future, besides (3.26), (3.27) we shall need the following estimate for the solution (3.11)

$$
\|u\|_{H^{4}(\Omega)} \leq C_{3}\|v\|_{H^{2}(\Omega)} .
$$

Now, we are able to state the result of convergence of the iterative process, constructed in the subsection 3.1.

Below, we assume that $f \in L_{2}(\Omega), g \in H^{3 / 2}(\Gamma)$ and $h \in H^{7 / 2}(\Gamma)$. Then BVP (1.1)-(1.3) has an unique solution $u \in H^{4}(\Omega)$ (see [10]). Here, it is recalled that $H^{s}(\Omega)$ and $H^{s}(\Gamma)$ are the Sobolev spaces.

Theorem 1. Let $\gamma_{1}$ and $\gamma_{2}$ be the lower and upper bounds of the spectrum of the operator B, i.e.

$$
\gamma_{1} I \leq B \leq \gamma_{2} I
$$

where $I$ is the identity operator in $L_{2}(\Omega)$.

Let $u^{*}$ be the solution of Problem (1.1)-(1.9) and $D^{*}$ be the solution of Equation (3.18).

Then, if $\left\{\tau_{k+1}\right\}$ is the Chebyshev collection of parameters, constructed by the bounds $\gamma_{1}$ and $\gamma_{2}$, namely

$$
\begin{gathered}
\tau_{0}=\frac{2}{\gamma_{1}+\gamma_{2}}, \tau_{k}=\frac{\tau_{0}}{\rho_{0} t_{k}+1}, t_{k}=\cos \frac{2 k-1}{2 M} \pi, k=1,2, \ldots, M \\
\rho_{0}=\frac{1-\xi}{1+\xi}, \quad \xi=\frac{\gamma_{1}}{\gamma_{2}}
\end{gathered}
$$


we have

$$
\left\|u^{(M)}-u^{*}\right\|_{H^{4}(\Omega)} \leq C q_{M}
$$

where

$$
C=\sqrt{C_{1}} C_{3}\left\|D^{(0)}-D^{*}\right\|_{L_{2}(\Omega)},
$$

$C_{1}, C_{3}$ being the constants in (3.26), (3.29), respectively

$$
q_{M}=\frac{2 \rho_{1}{ }^{M}}{1+\rho_{1}^{2 M}}, \rho_{1}=\frac{1-\sqrt{\xi}}{1+\sqrt{\xi}} .
$$

In the case of the stationary iterative process, $\tau_{k}=\tau_{0}(k=1,2, \ldots)$ we have

$$
\left\|u^{(k)}-u^{*}\right\|_{H^{4}(\Omega)} \leq \rho_{0}^{k}\left\|D^{(0)}-D^{*}\right\|_{L_{2}(\Omega)}, k=1,2, \ldots
$$

Proof. According to the general theory of the two-layer iterative schemes (see $[12,13])$ for the operator equation (3.18) we have

$$
\left\|D^{(M)}-D^{*}\right\|_{L_{2}(\Omega)} \leq q_{M}\left\|D^{(0)}-D^{*}\right\|_{L_{2}(\Omega)}
$$

if the parameter $\left\{\tau_{k+1}\right\}$ is chosen by the formula (3.31) and

$$
\left\|D^{(k)}-D^{*}\right\|_{H^{4}(\Omega)} \leq \rho_{0}{ }^{k}\left\|D^{(0)}-D^{*}\right\|_{L_{2}(\Omega)}, \quad k=1,2, \ldots
$$

if $\tau_{k}=\tau_{0}(k=1,2, \ldots)$.

The corresponding estimates (3.32) and (3.35) follow from (3.26), (3.29) applied to the problems

$$
\begin{gathered}
\Delta\left(v^{(k)}-v^{*}\right)-a\left(v^{(k)}-v^{*}\right)=D^{(k)}-D^{*}, x \in \Omega, \\
\left.\left(v^{(k)}-v^{*}\right)\right|_{\Gamma}=0, \\
\Delta\left(u^{(k)}-u^{*}\right)=v^{(k)}-v^{*}, x \in \Omega, \\
\left.\left(u^{(k)}-u^{*}\right)\right|_{\Gamma}=0,
\end{gathered}
$$

which are obtained in the result of the subtraction (3.3) and (3.4) from (3.5) and (3.6), respectively. Here, $u^{*}, v^{*}$ and $D^{*}$ satisfy (3.3) and (3.4).

\section{CONCLUDING REMARK}

The determination of the lower and upper bounds $\gamma_{1}$ and $\gamma_{2}$ of the spectrum of the operator $B$ is a difficult problem to be researched. Nevertheless, for the coarse estimates we can choose $\gamma_{1}=1$, $\gamma_{2}=1+C_{2}+a C_{1} C_{2}$, where $C_{1}, C_{2}$ are the constants in (3.26), (3.27). But in their turn, the constants $C_{1}, C_{2}$ are not easy to find, too. Therefore, while there is not any information of $\gamma_{1}$ and $\gamma_{2}$, we propose to use the simple iteration method with $0<\tau<2 /\|B\|$, for example, $0<\tau<2$ because $\|B\|>1$. Then the iterative process will still converge, but we can not obtain any estimate of the convergence rate of the method (see [13], p. 105-106). 


\section{REFERENCES}

[1] A. A. Abramov and V.I. Ulijanova, On a method for solving biharmonic type equation with singularly small parameter, Journal of Comput. Math. and Math. Physics 32 (4) (1992) 567575 (Russian).

[2] D. Begis, A. Perronet, The Club Modules, In book Numerical Methods in Applied Mathematics, Eds. G. Marchuk and J. L. Lions, Nauka, Novosibirsk, 1982, 212-236 (Russian).

[3] Dang Quang A, On an iterative method for solving a boundary value problem for fourth order differential equation, Math. Physics and Nonlinear Mechanics 10 (44) (1988) 54-59 (Russian).

[4] Dang Quang A, Approximate method for solving an elliptic problem with discontinuous coefficients, Journal of Comput. and Appl. Math. 51 (2) (1994) 193-203.

[5] Dang Quang A, Boundary operator method for approximate solution of biharmonic type equation, Journal of Math. 22 (1-2) (1994) 114-120.

[6] Dang Quang A, Mixed boundary-domain operator in approximate solution of biharmonic type equation, Vietnam Journal of Math. 26 (3) (1998) 249-258.

[7] N. Drenska, Numerical method for solving an elliptic equation of fourth order, Cepguka: Bul. mat. cnuc. 15 (2) (1989) 108-113; (Abstract in Referative Journal Mathematics, 2/1990, $2 \Gamma 200)$.

[8] A. Dorodnisyn, N. Meller, On some approaches to the solution of the stationary Navier-Stock equation, Journal of Comp. Math. and Math. Physics 8 (2) (1968) 393-402 (Russian).

[9] R. Glowinski, J. L. Lions, and R. Tremolière, Analyse Numérique des Inequations Variationelles, Dunod, Paris, 1976.

[10] J. L. Lions and E. Magenes, Problèmes aux Limites Non Homogènes et Applications, 1, Dunod, Paris, 1968.

[11] B. V. Palsev, On the expansion of the Dirichlet problem and a mixed problem for biharmonic equation into a series of decomposed problems, Journal of Comput. Math. and Math. Physics 6 (1) (1966) 43-51 (Russian).

[12] A. Samarskij and E. Nikolaev. Numerical Methods for Grid Equations, 2, Birkhauser, Basel, 1989.

[13] A. Samarskij, Introduction to Numerical Methods, Moscow, Nauka, 1988.

[14] R. Szilard, Theory and Analysis of Plates, Prentice-Hall, 1974.

Tóm tăt. Việc giải các bài toán biên đối với phương trình cấp bốn bằng cách đưa chúng về các bài toán biên đối với các phương trình cấp hai với mục đích sử dụng các thành tựu đã đạt được đối với các phương trình này thu hút sự quan tâm cưa nhiều nhà nghiên cứu.

Trong bài báo này, nhờ kỹ thuật được phát triển trong các công trình gần đây của mình, chúng tôi đã xây dựng được các phương pháp lặp giải bài toán biên thứ hai đối với phương trình loại song điều hòa. Tốc độ hội tụ của phương pháp được thiết lập.

Institute of Information Technology

Received: May 18, 1998

National Centre for Natural Sciences and Technology of Vietnam. 